\title{
BRAGG REFLECTION BAND STOP FILTER FOR ECE ON WEGA
}

\author{
D. Wagner ${ }^{1}$, W. Kasparek ${ }^{2}$, F. Leuterer ${ }^{1}$, F. Monaco ${ }^{1}$, M. Münich ${ }^{1}$, \\ H. Schütz ${ }^{1}$, T. Stange ${ }^{4}$, J. Stober ${ }^{1}$, M. Thumm ${ }^{3}$ \\ ${ }^{I}$ Max-Planck-Institut für Plasmaphysik, EURATOM-IPP, Boltzmannstr.2, \\ D-85748 Garching, Germany \\ e-mail: dietmar.wagner@ipp.mpg.de \\ ${ }^{2}$ Institut für Plasmaforschung, Universität Stuttgart, Pfaffenwaldring 31, \\ D-70569 Stuttgart, Germany \\ ${ }^{3}$ Karlsruhe Institute of Technology, EURATOM-KIT \\ Institut fürHochleistungsimpuls- und Mikrowellentechnik, \\ PO Box 3640, D-76021 Karlsruhe, Germany \\ ${ }^{4}$ Max-Planck-Institut für Plasmaphysik, Teilinstitut Greifswald, EURATOM-IPP, \\ Wendelsteinstr.1,D-17491 Greifswald, Germany
}

Received 7 July 2011

\begin{abstract}
Notch filters are required to protect sensitive millimeter wave diagnostics in fusion experiments from gyrotron stray radiation in the plasma vessel. A new type of notch filter based on a waveguide Bragg reflector is presented that can provide one or more defined stop bands in one standard waveguide frequency band.
\end{abstract}

Key words: Electron cyclotron resonance heating, gyrotron, millimeter wave diagnostics, notch filter. 


\section{Introduction}

Modern Electron Cyclotron Resonance Heating (ECRH) systems at thermonuclear fusion experiments operate at one or more frequencies in the millimeter wave range at power levels of several megawatts and pulse lengths from several seconds to continuous $(\mathrm{cw})$. The gyrotron sources used on these experiments operate at a single frequency or at several frequencies using frequency step-tunable gyrotrons. Sensitive millimeter-wave diagnostic systems need to be protected against ECRH stray radiation [1]. In addition, a specific problem concerning notch filters is the frequency drift of high power gyrotrons specifically at the beginning of the pulse [2]. This frequency drift also varies for different gyrotrons from several tens up to hundreds of $\mathrm{MHz}$. A typical frequency specification for the operating frequency of high-power gyrotrons is $f_{\text {gyr }} \pm 500 \mathrm{MHz}$. This means that the stop bands of the notch filters have to be rather wide, especially for systems employing a number of different gyrotrons. For multi-frequency systems several defined stop bands are required. Both requirements are difficult to cope with using the available filter technology. A Bragg reflector in an oversized circular waveguide section can provide both, steep frequency slopes and a stop band with defined width. By applying higher order Bragg resonances it can also provide several defined stop bands within one standard waveguide frequency band. Another advantage of this approach is a very small insertion loss due to the low ohmic attenuation in the oversized waveguide section outside of the stop band of the filter.

A first prototype of a notch filter based on Bragg reflection of high order modes in circular waveguide cross section has been built for the Electron Cyclotron Emission (ECE) diagnostic system at the WEGA stellarator in Greifswald, Germany. At WEGA a $28 \mathrm{GHz}, 10 \mathrm{~kW}$, cw gyrotron is in operation for Electron Bernstein Wave heating experiments [3]. The sensitive ECE diagnostic system has to be protected against stray radiation especially in cases with non-perfect millimeter wave absorption in the plasma.

\section{Bragg Reflector in Oversized Circular Waveguide}

In a cylindrical waveguide with periodical azimuthal radial grooves (Fig.1) a propagating waveguide mode can be reflected into itself or into modes with different radial indices if the corrugation period $\mathrm{p}$ satisfies the Bragg condition $[4,5]$ :

$$
p=\lambda_{\text {Bragg }}=\frac{2 \pi}{k_{\text {Bragg }}}=\frac{2 \pi}{k_{z 1}+k_{z 2}},
$$

where $\mathrm{k}_{\mathrm{z} 1}$ and $\mathrm{k}_{\mathrm{z} 2}$ are the longitudinal wave numbers of the incident and reflected modes respectively. 

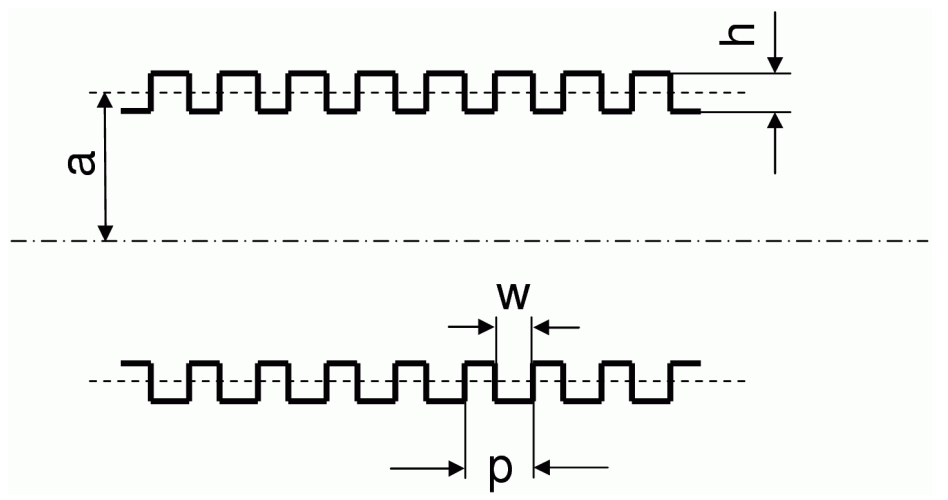

Fig.1 Cylindrical waveguide geometry with constant corrugation with period $p$, width $\mathrm{w}$ and depth $\mathrm{h}$.

Inserting the dispersion relation for circular waveguides into equation (1) gives the frequencies where the incident $\mathrm{TE}_{11}$ mode in a corrugated circular waveguide with a corrugation period of $\mathrm{p}=\lambda_{\text {Bragg }}$ couples to other reflected modes:

$$
f_{1 n}=\frac{c \cdot \lambda_{\text {Bragg }}}{8 \pi^{2}} \cdot \sqrt{\left(\frac{4 \pi^{2}}{\lambda_{\text {Bragg }}^{2}}+\frac{x_{11}^{2}+x_{1 n}^{2}}{a^{2}}\right)^{2}-\frac{4 \cdot x_{11}^{2} \cdot x_{1 n}^{2}}{a^{4}}},
$$

where $\mathrm{c}$ is the velocity of light, $\mathrm{a}$ is the waveguide radius, $\mathrm{x}_{11}$ and $\mathrm{x}_{1 \mathrm{n}}$ are the eigenvalues of the incident $\mathrm{TE}_{11}$ mode and the reflected $\mathrm{TE}_{1 \mathrm{n}}$ and $\mathrm{TM}_{1 \mathrm{n}}$ modes. The dispersion diagram for a Bragg reflector with a constant period of $\lambda_{\text {Bragg }}=5.27 \mathrm{~mm}$ in a waveguide with inner diameter (I.D.) of $27.79 \mathrm{~mm}$ for the incident fundamental $\mathrm{TE}_{11}$ mode is given in Fig.2. The resonant frequencies where $\mathrm{k}_{\mathrm{z} 2}=\mathrm{k}_{\mathrm{Bragg}}-\mathrm{k}_{\mathrm{z} 1}$ are indicated by the rectangles. Since the Bragg reflector can be composed by a series of radial step discontinuities, an accurate method to calculate the reflectivity of this structure is the scattering matrix formalism where both propagating and evanescent modes are taken into account $[6,7]$. The spectral width of the Bragg reflection depends on the number of periods $\mathrm{N}$, corrugation amplitude $\mathrm{h}$ and aspect ratio $\mathrm{w} / \mathrm{p}$, where $\mathrm{w}$ is the width of the grooves. However, the amplitude is typically limited to $h<0.05 \cdot \lambda_{0}$, where $\lambda_{0}$ is the free space wavelength, if a high mode purity of the reflected mode in the oversized waveguide is required [6]. In case of higher order Bragg resonances there is also a large difference between $\mathrm{TE}_{1 \mathrm{n}}$ and $\mathrm{TM}_{1 \mathrm{n}}$ mode coupling. For higher order TM modes the magnitude of the azimuthal magnetic field component at the waveguide wall remains almost constant and so does the coupling factor [8], whereas the coupling of the fundamental $\mathrm{TE}_{11}$ mode to $\mathrm{TE}_{1 \mathrm{n}}$ modes with higher radial indices reduces with increasing mode index $\mathrm{n}$ and therefore increasing eigenvalue (zero of the derivative of the Bessel 
function of first order). As an example, the calculated reflection as a function of frequency for a Bragg reflector with 200 periods $\left(\mathrm{p}=\lambda_{\text {Bragg }}=5.27 \mathrm{~mm}\right.$ ) of rectangular corrugation with a constant corrugation amplitude of $0.25 \mathrm{~mm}$ in a circular waveguide with I.D. $=27.79 \mathrm{~mm}$ is given in Fig.3. Tapering of the corrugation amplitude profile strongly reduces resonance overlaps and side bands in the frequency response [6,7].

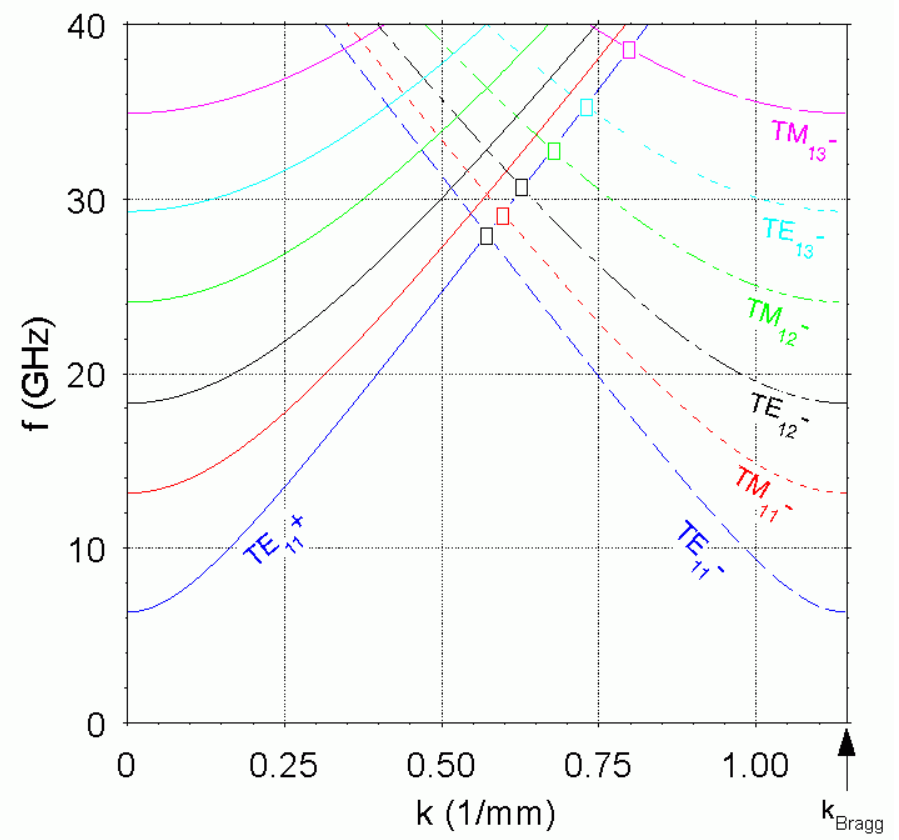

Fig.2 Dispersion diagram of a cylindrical waveguide with I.D. $=27.79 \mathrm{~mm}$ constant corrugation with $\mathrm{k}_{\text {Bragg }}=2 \pi / \lambda_{\text {Bragg }}=1.19 \mathrm{~mm}^{-1}\left(\lambda_{\text {Bragg }}=5.27 \mathrm{~mm}\right)$. 


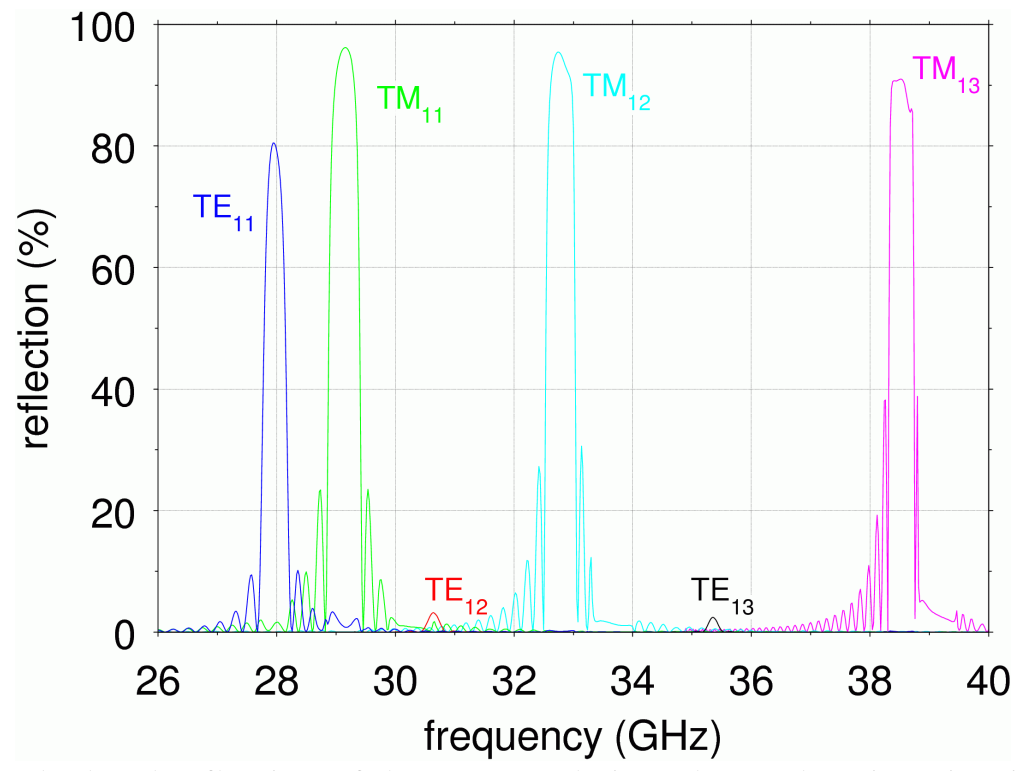

Fig. 3 Calculated reflection of the $\mathrm{TE}_{11}$ mode into the modes given in Fig.2 for a Bragg reflector with constant rectangular corrugation (I.D. $=27.79 \mathrm{~mm}$, $\lambda_{\text {Bragg }}=5.27 \mathrm{~mm}, \mathrm{~h}=0.25 \mathrm{~mm}$ ).

\section{Notch Filter Principle}

In order to realize a notch filter with one or more specific stop bands and wide pass bands with low insertion loss, a Bragg reflector that reflects the incident fundamental $\mathrm{TE}_{11}$ into a $\mathrm{TM}_{1 \mathrm{n}}$ mode close to cutoff is combined with two waveguide tapers to fundamental waveguide diameter (Fig.4). Here the fundamental $\mathrm{TE}_{11}$ mode is the only propagating mode at both ends of the reflector. The incident $\mathrm{TE}_{11}$ mode couples through the taper and is converted to the backward propagating high order $\mathrm{TM}_{1 \mathrm{n}}$ mode by the Bragg structure if equation (1) is satisfied. This $\mathrm{TM}_{1 \mathrm{n}}$ mode is trapped in the oversized waveguide section by the tapers. Once reflected at the input taper it will propagate in forward direction again and be converted back into the backward propagating $\mathrm{TE}_{11}$ mode which then can pass through the taper. Therefore at higher order Bragg resonances, the filter acts as a reflector for the incoming $\mathrm{TE}_{11}$ mode. Outside of the Bragg resonances, when equation (1) is not satisfied, the $\mathrm{TE}_{11}$ mode can propagate through the oversized waveguide structure with only very small ohmic attenuation compared to propagating in a fundamental waveguide. Coupling to other modes is negligible in the nonresonant case due to the small corrugation amplitude. 


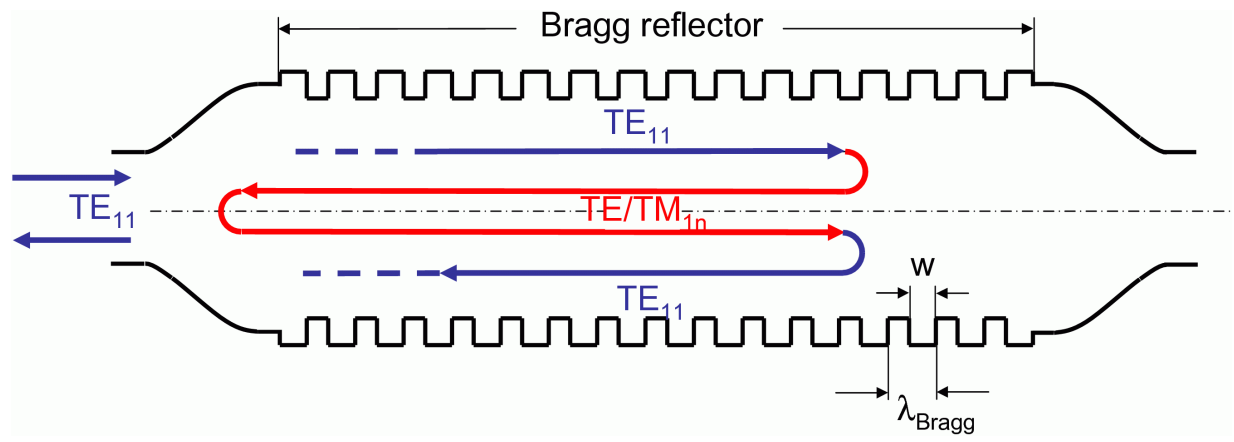

Fig.4 Principle scheme of the notch filter.

\section{Bragg Reflector Design}

A Bragg reflector was designed for the $\mathrm{TE}_{11}-\mathrm{TM}_{12}$ resonance at $28 \mathrm{GHz}$ in a cylindrical waveguide with I.D. $=27.79 \mathrm{~mm}$ (Table 1). The reason to choose this waveguide diameter was to make use of available hardware components of the ECE transmission line at WEGA with the same diameter. The input and output sections of the Bragg reflector have a tapered corrugation profile (Fig.5) to ensure high mode purity and minimum side bands in the frequency response. The length of the tapered section corresponds to 50 corrugation periods at both ends of the Bragg reflector with a profile given by:

$$
h(z)=h_{0} \cdot \frac{\sin \left(\pi \cdot\left(1-\frac{z}{z_{0}}\right)\right)}{\pi \cdot\left(1-\frac{z}{z_{0}}\right)},
$$

where $\mathrm{h}_{0}$ is the maximum corrugation amplitude, $\mathrm{z}_{0}$ is the total length of the tapered section of the corrugation .

The corrugations in the middle of the Bragg reflector have a constant depth of $\mathrm{h}_{0}$. The corrugation of the complete Bragg reflector is plotted in Fig.6. The calculated reflection and transmission of this corrugated waveguide structure is given in figures 7 and 8 . There are 3 Bragg resonances in this frequency band for the given waveguide diameter $\left(\mathrm{TM}_{12}, \mathrm{TE}_{13}\right.$ and $\left.\mathrm{TM}_{13}\right)$. The mode purity of the calculated reflection signal is very high (Fig.7). There is some mode conversion, mainly into the $\mathrm{TM}_{11}$ mode, in forward direction (Fig.8).

\begin{tabular}{|c|c|c|c|c|}
\hline I.D. $(\mathrm{mm})$ & $\mathrm{N}$ & $\lambda_{\text {Bragg }}(\mathrm{mm})$ & $\mathrm{h}_{0}(\mathrm{~mm})$ & $\mathrm{w} / \lambda_{\text {Bragg }}$ \\
\hline 27.79 & 300 & 7.2 & 0.4 & 0.5 \\
\hline
\end{tabular}

Table 1: Geometry of the Ka-band Bragg reflector (I.D., number of corrugation periods $\mathrm{N}$, period $\mathrm{p}$ and maximum corrugation amplitude $\mathrm{h}_{0}$ ). 


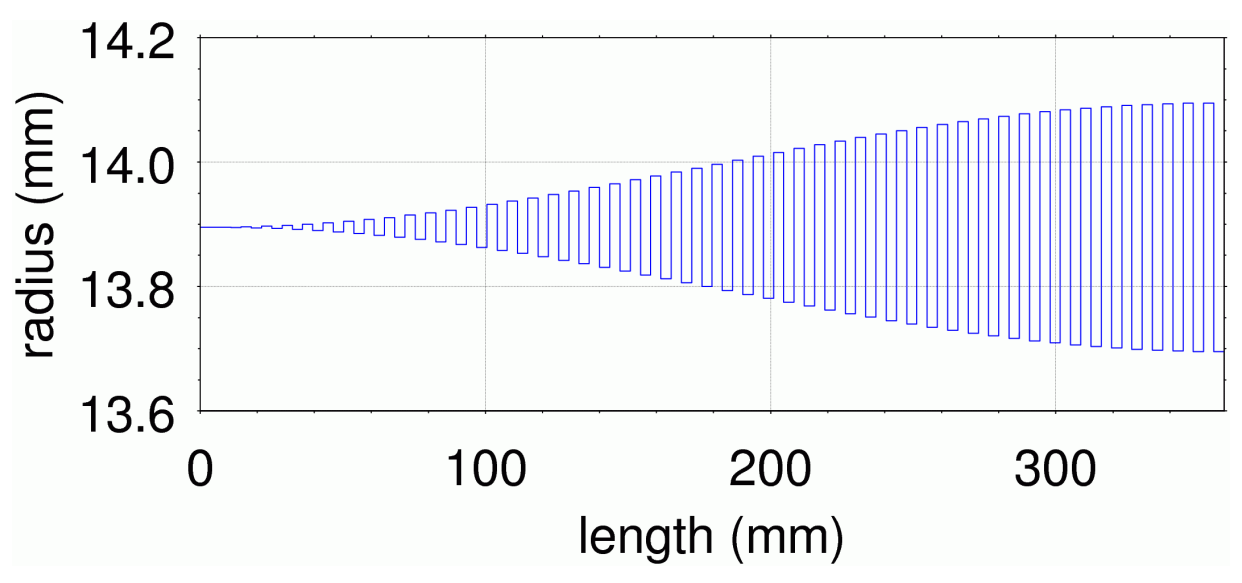

Fig.5 Tapered corrugation profile at the input of the $28 \mathrm{GHz}$ Bragg reflector.

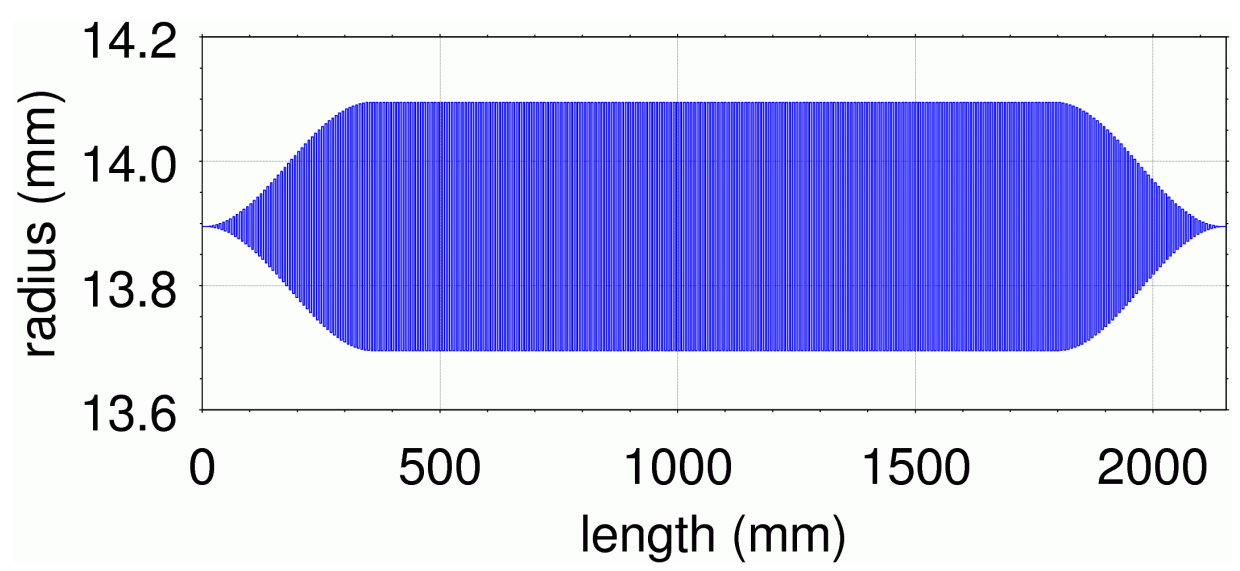

Fig.6 Corrugation profile of the $28 \mathrm{GHz}$ Bragg reflector. 


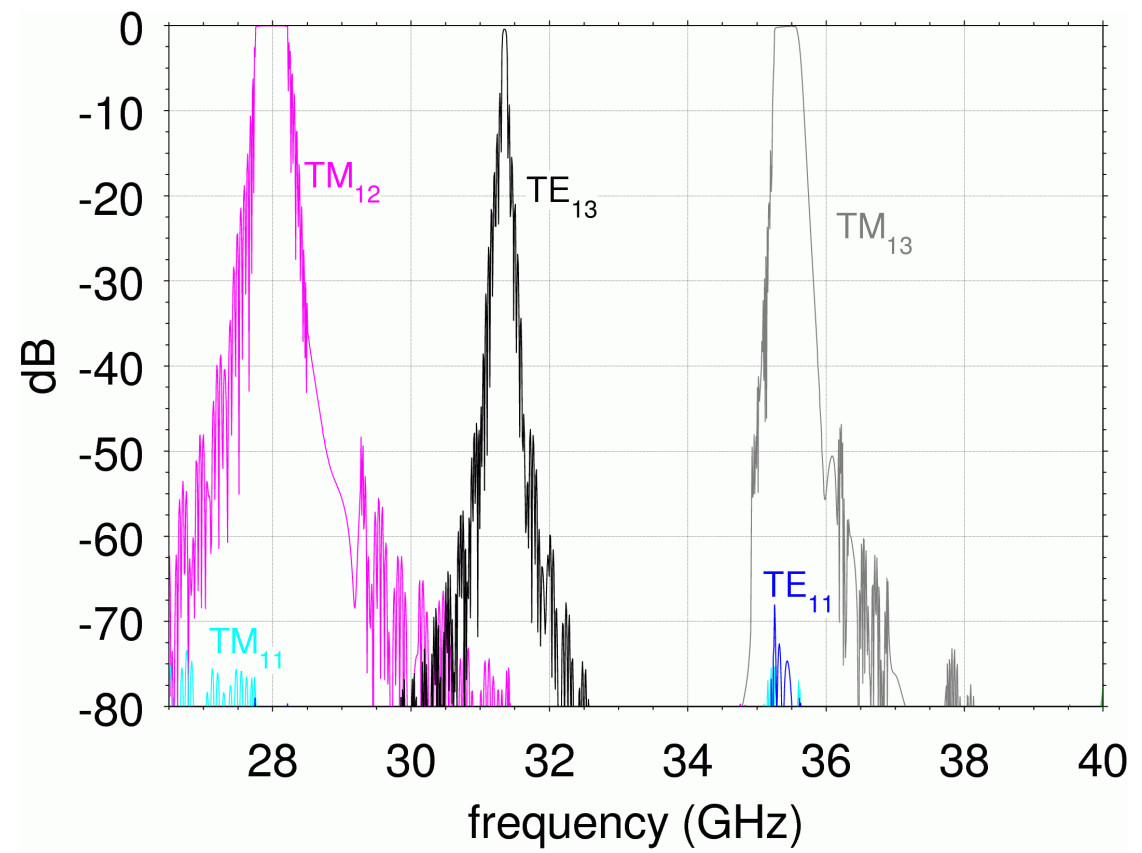

Fig.7 Calculated reflection of the Bragg reflector given in Fig. 6 for the incident $\mathrm{TE}_{11}$ mode.

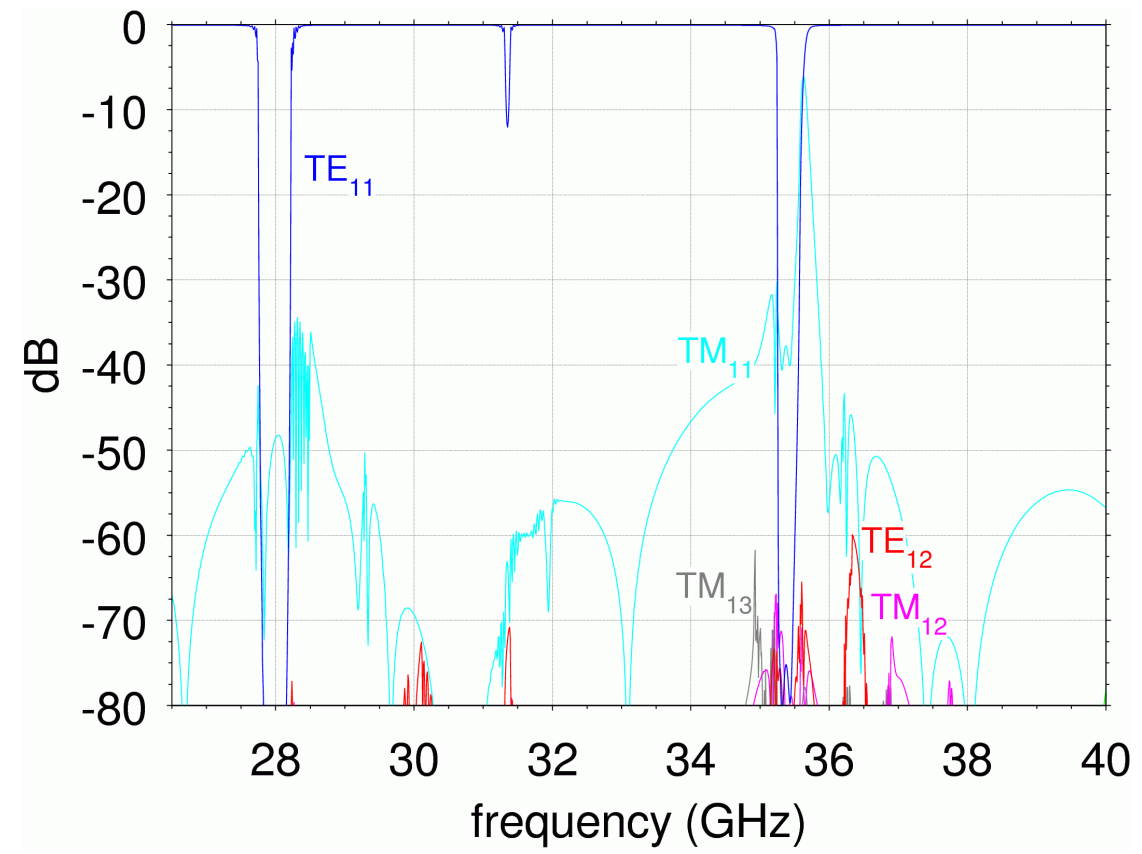

Fig.8 Calculated transmission of the Bragg reflector given in Fig.6 for an incident $\mathrm{TE}_{11}$ mode. 


\section{Nonlinear Taper}

The transition from a standard circular waveguide (I.D. $=8 \mathrm{~mm}$ ) to the diameter of $27.79 \mathrm{~mm}$ of the Bragg reflector section is provided by a smooth-wall nonlinear taper [8] as shown in Fig.9. The taper was modelled by a staircase representation and calculated using the scattering matrix formalism. It was optimized to couple less than $-50 \mathrm{~dB}$ of power to other modes than the incident fundamental $\mathrm{TE}_{11}$ mode at its output. The total length of the taper is $421 \mathrm{~mm}$. The calculated reflected and transmitted mode spectra of this taper alone as a function of frequency are given in figures 10 and 11.

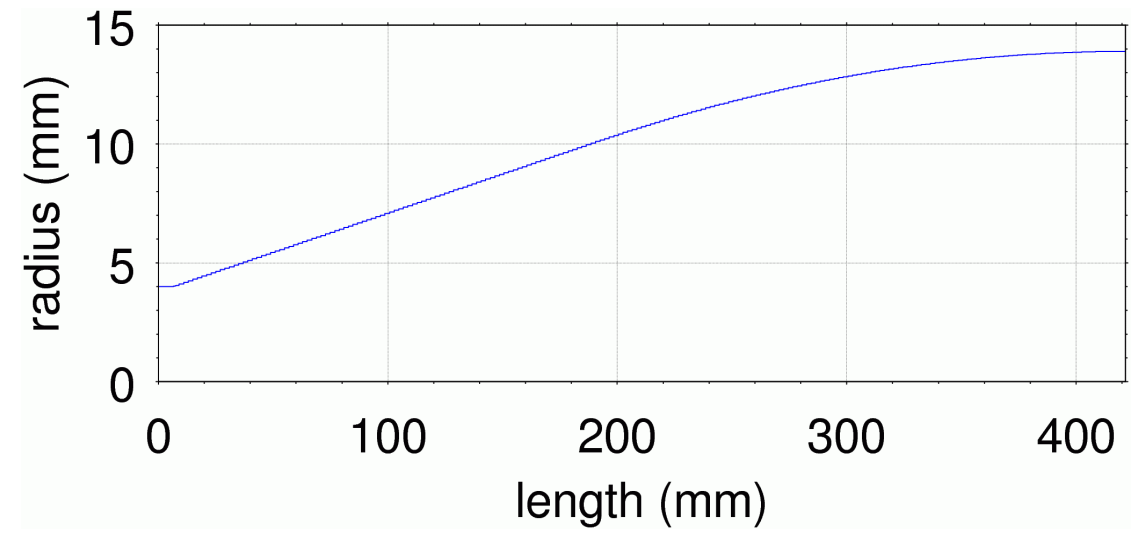

Fig.9 Nonlinear taper from I.D. $=8 \mathrm{~mm}$ to $27.79 \mathrm{~mm}$. 


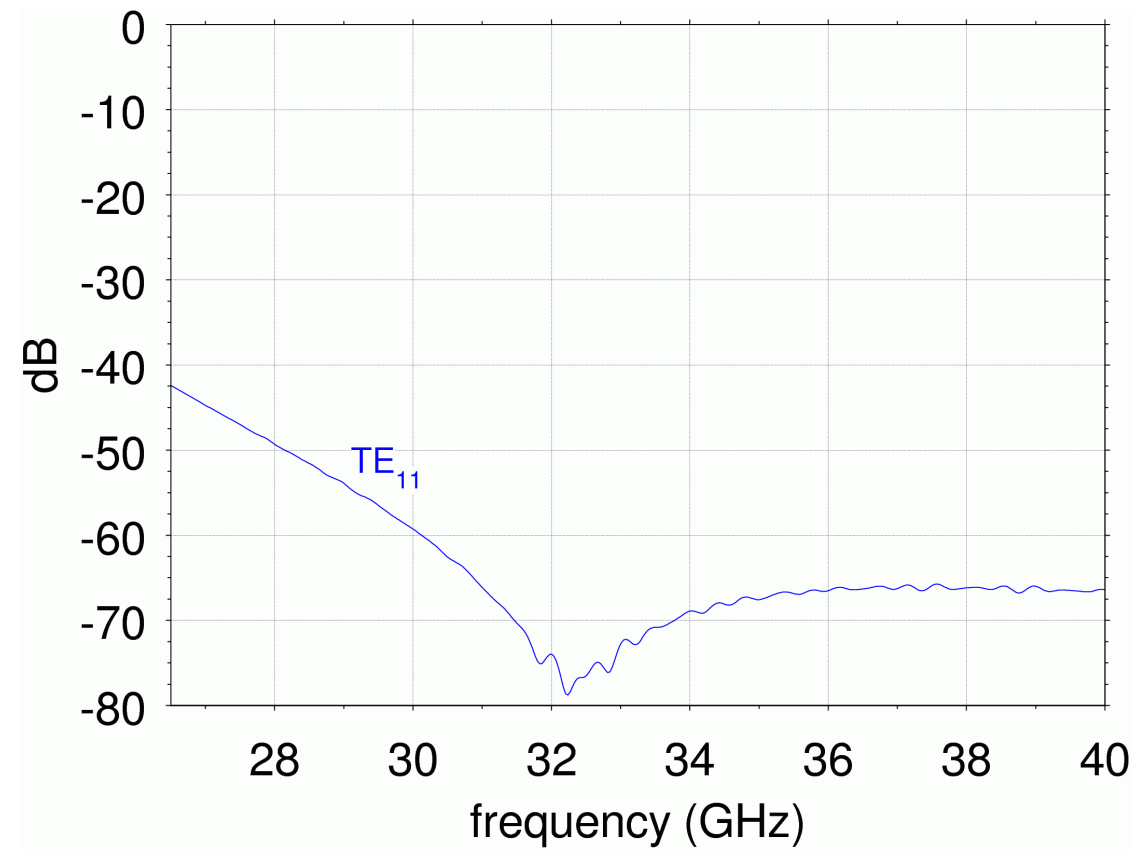

Fig.10 Calculated reflection of the waveguide taper given in Fig.9.

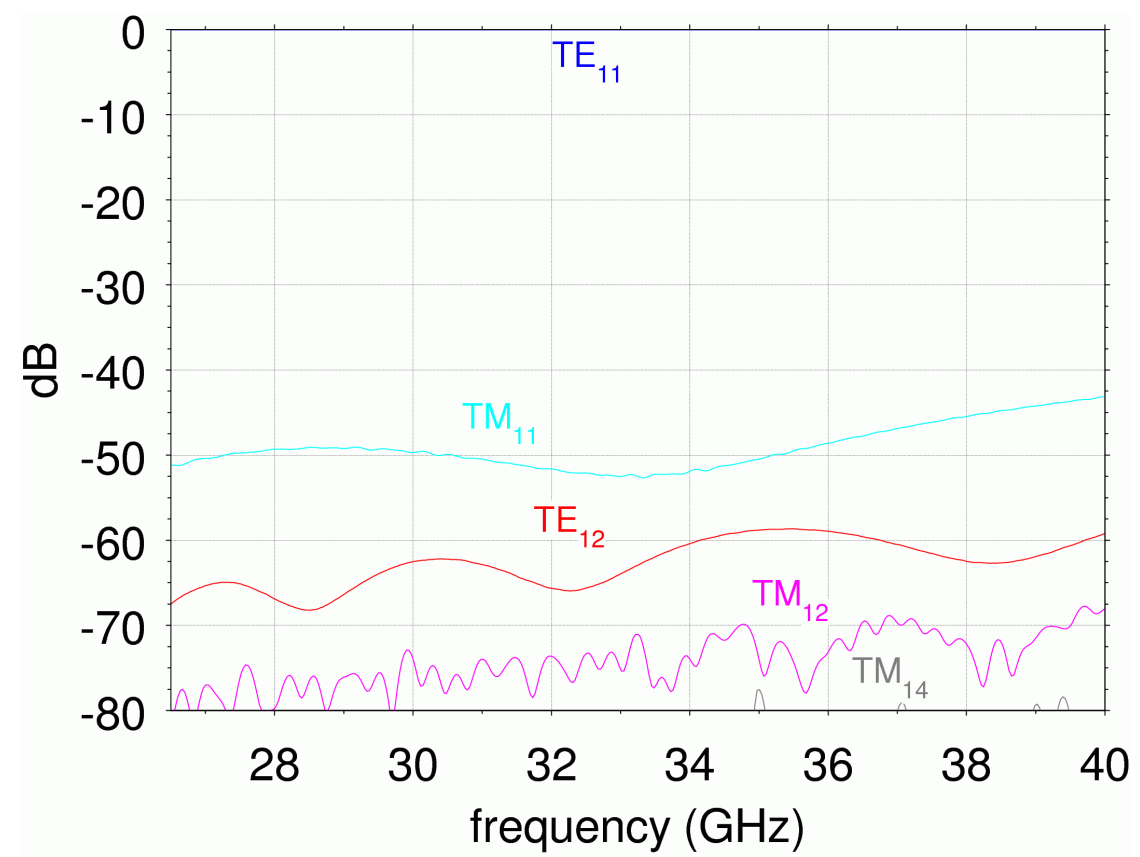

Fig.11 Calculated transmission of the waveguide taper given in Fig.9. 


\section{WEGA Band Stop Filter}

The total length of the notch filter including tapers is 3m (Fig.12). This corresponds to the distance between the ECE antenna at the WEGA experiment and the ECE receiver. Outside of the Bragg resonances the filter acts as a low loss transmission line for the ECE signal. A comparison between calculated and measured frequency response of the notch filter is given in Fig.13. The $\mathrm{TM}_{12}$ resonance is plotted in higher resolution in figure 14. The measured transmission function is in good agreement with theory. The measured and calculated insertion loss in between the stop bands is $-0.2 \mathrm{~dB}$.

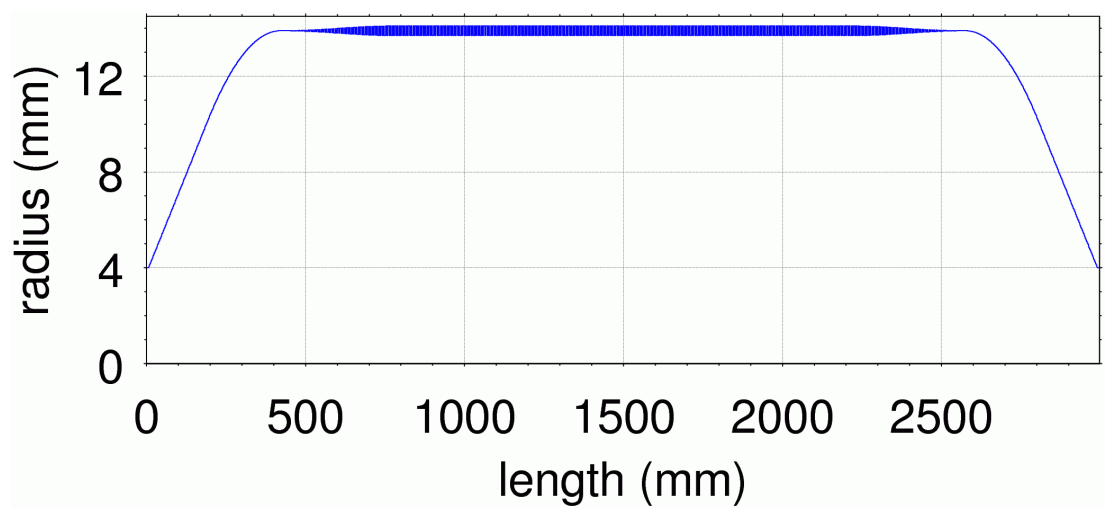

Fig.12 Geometry of the Ka-band notch filter.

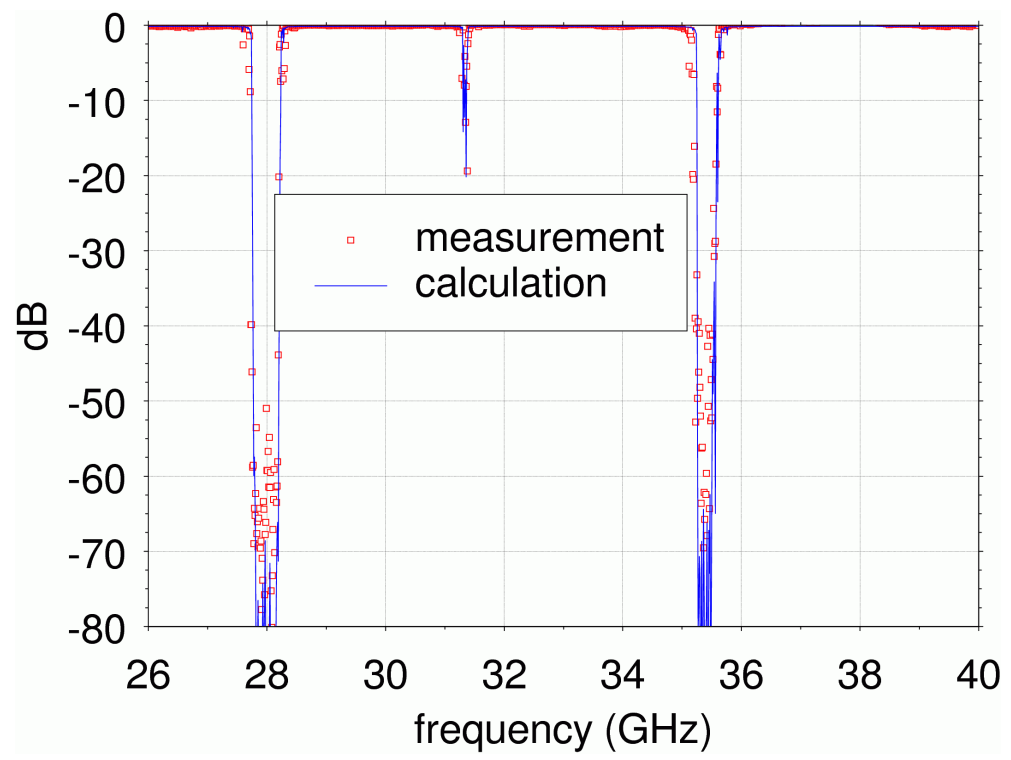

Fig.13 Comparison between calculated and measured transmission of the Kaband notch filter. 


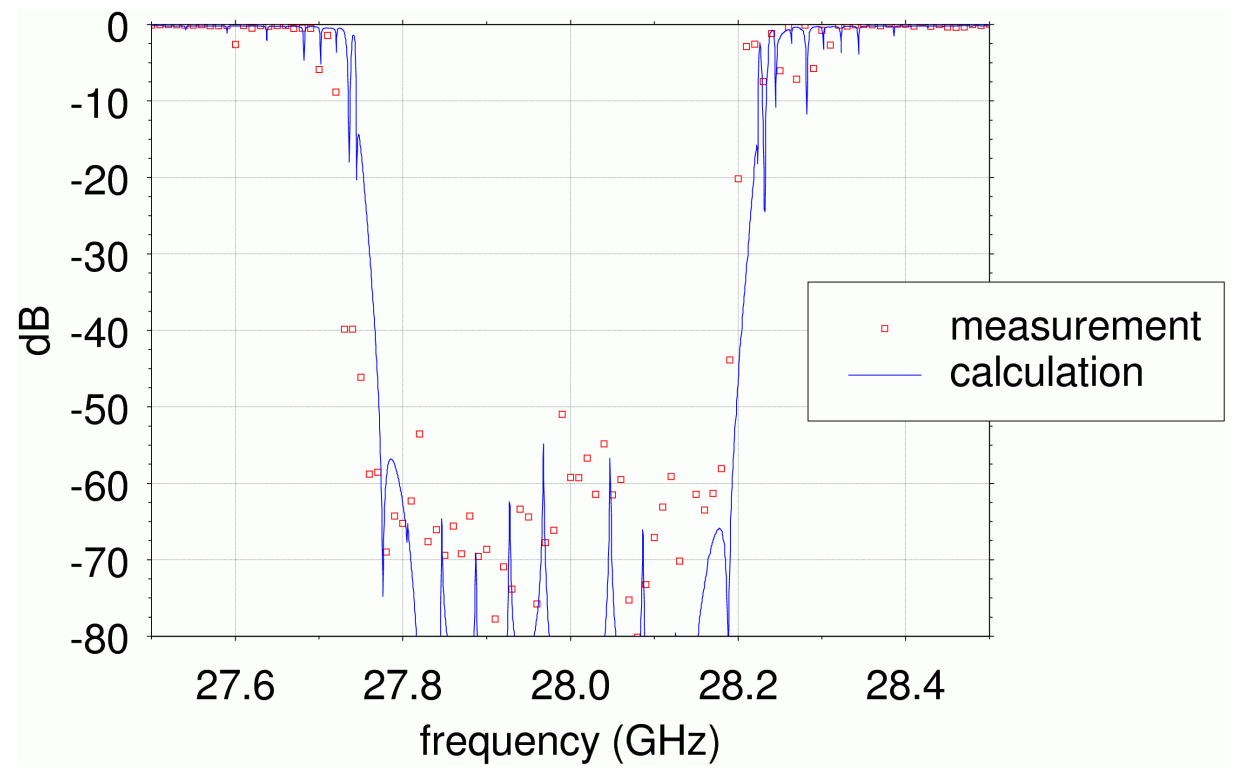

Fig.14 Comparison between calculated and measured transmission of the Kaband notch filter around $28 \mathrm{GHz}$. 


\section{Summary and Outlook}

A band stop filter based on a Bragg reflector in an oversized circular waveguide has been built and tested. The filter will be applied as a protection for the ECE diagnostic system at the WEGA stellarator at IPP Greifswald. The number of stop bands and their center frequencies depend on both, the corrugation period and the waveguide radius. This type of notch filter is therefore also an interesting option for fusion experiments where multifrequency ECRH systems are in use.

\section{References}

[1] P. Woskov, "Notch Filter Options for ITER Stray Gyrotron Radiation", Proceedings 49th Annual Meeting of the Division of Plasma Physics, Orlando, Florida, 52, No.11, NP8.00111, 2007.

[2] D. Wagner et al., "Status of the new multi-frequency ECRH System for ASDEX Upgrade", Nuclear Fusion, 48, 054006 (6pp), 2008.

[3] C.B. Warr et al., "New $28 \mathrm{GHz}$ Plasma ECR Heating System for the WEGA Stellarator, 33rd EPS Conference on Plasma Phys., Vol.30I, Rome, Italy, P-2.122, 2006.

[4] V.L. Bratman et al.., "FEL's with Bragg reflection resonators: Cyclotron autoresonance masers versus Ubitron", IEEE J. Quantum Electronics, 19, no. 3, 282-293, 1983.

[5] N.S. Ginzburg et al., "Improving selectivity of free electron maser with 1D Bragg resonator using coupling of propagating and trapped waves", Phys. Rev. ST-AB, Vol.8, p.040705, 2005.

[6] D. Wagner et al., "Bragg Reflector Design in Highly Oversized Waveguides", Proceedings $16^{\text {th }}$ Int. Conf. on Infrared and Millimeter Waves, Lausanne, Switzerland, 537-539, 1991.

[7] C.K. Chong et al., "Bragg reflectors", IEEE Trans. on Plasma Science, 20, No.3, 393-402, 1992.

[8] Katsenelenbaum et al., "Theory of nonuniform waveguides - the cross section method", IEE Electromagn. Waves Series, 44, 1998. 